\title{
1 Thermo-responsive, self-assembling biointerface for on demand release of \\ 2 surface-immobilised proteins
}

3

4

5

6

7

8

9

10

\section{Angela Saccardo, ${ }^{a}$ Mikhail Soloviev ${ }^{\mathrm{b}}$ and Enrico Ferrari ${ }^{* a}$}

a. School of Life Sciences, University of Lincoln, Lincoln LN6 7TS, UK.

b. Department of Biological Sciences, Royal Holloway University of London, Egham TW20 OEX, UK.

*Address correspondence to eferrari@lincoln.ac.uk

Published in Biomaterials Science, 2020,8, 2673-2681, https://doi.org/10.1039/C9BM01957J

Submitted: 05 Dec 2019. Accepted:01 Apr 2020. First published: 01 Apr 2020

\section{Abstract}

Dedicated chemistries for on-demand capture and release of biomolecules at the solid-liquid interface are required for applications in drug delivery, for the synthesis of switchable surfaces used in analytical devices and for the assembly of next-generation biomaterials with complex architectures and functions. Here we report the engineering of a binary self-assembling polypeptide system for reversible protein capture, immobilisation and controlled thermo-responsive release from a solid surface. The first element of the binary system is a universal protein substrate immobilised on a solid surface. This protein is bio-inspired by the neuronal SNAP25, which is the protein involved in the docking and fusion of synaptic vesicles to the synaptic membrane. The second element is an artificial chimeric protein engineered to include distinct domains from three different proteins: Syntaxin, VAMP and SNAP25. These native proteins constitute the machinery dedicated to vesicle trafficking in eukaryotes. We removed approximately $70 \%$ of native protein sequence from these proteins and constructed a protein chimera capable of high affinity interaction and self-assembly with immobilised substrate. The interaction of the two parts of the engineered protein complex is strong but fullyreversible and therefore the chimera can be recombinantly fused as a tag to a protein of interest, to allow spontaneous assembly and stimuli-sensitive release from the surface upon heating at a predetermined temperature. Two thermo-responsive tags are reported: the first presents remarkable thermal stability with melting temperature of the order of $80^{\circ} \mathrm{C}$; the second disassembles at a substantially lower temperature of about $45^{\circ} \mathrm{C}$. The latter is a promising candidate for remotecontrolled localised delivery of therapeutic proteins, as physiologically tolerable local increase of temperatures in the $40-45^{\circ} \mathrm{C}$ range can be achieved using magnetic fields, infra-red light or focused ultrasound. Importantly, these two novel polypeptides provide a broader blueprint for the engineering of future functional proteins with predictable folding and response to external stimuli. 
Controlled release is increasingly studied in the context of protein therapeutics, for which spatiotemporal- and dosage-controlled delivery would be desirable. ${ }^{1}$ Controlled release of biologics such as recombinant proteins would be particularly beneficial to enhance their therapeutic efficacy, as they often present limited half-life and immunogenicity issues. Although improving the therapeutic window can be achieved by a slow and prolonged release of drugs using passive delivery systems, a triggerable drug delivery system that can respond to stimuli could control drug release more effectively. ${ }^{2}$ To address this, many remotely triggerable and stimuli-responsive materials have been developed for the design of novel drug delivery systems. ${ }^{3,4}$ Stimuli-responsive interfaces have been engineered to respond to either physiological changes (e.g., $\mathrm{pH}$, redox potential, glucose concentration, specific enzymatic activity) or external stimuli, such as electric or magnetic fields, mechanical forces (e.g., ultrasound mediated), light and heat.

Thermo-responsive materials are particularly promising due to the range of technologies that are already available to increase local temperatures on demand: $i$. focused ultrasound-mediated cavitation of microbubbles can heat tissue above $56^{\circ} \mathrm{C}$ within seconds; ${ }^{2}$ ii. tissue-penetrating near infrared light can be used to deliver local heat via nanomaterial-mediated photothermal effect; ${ }^{5}$ iii. magnetic fields can be used to increase temperatures in the presence of iron oxide nanoparticles, like those used in magnetic resonance imaging. ${ }^{6}$ As a consequence of multiple ways to deliver controlled heat locally, thermo-responsive materials such as hydrogels and polymers presenting sharp phase transition temperatures have been synthesised and used to encapsulate or cross-link drugs, including therapeutic proteins. ${ }^{1}$ Although thermo-responsive interfaces have been described mostly for their potential use in drug delivery, biomaterials that respond to thermal stimuli have also been applied in other contexts. Examples include protein affinity purification, ${ }^{7}$ rapid diagnostic assays, ${ }^{8}$ remotelycontrolled protein pores, ${ }^{9}$ and remote control of enzymatic activity. ${ }^{10}$

To enable dynamic and reversible structural change or release of proteins from surfaces, several protein or peptide conformational switches have been studied. ${ }^{11}$ Bio-mimetic conformational switches have been engineered from natural systems, such as barnacle adhesive proteins-inspired self-assembling peptides, ${ }^{12}$ or de novo designed from canonical and predictable structural motifs such as $\alpha$-helical coiled-coils and $\beta$-hairpins. ${ }^{13}$ Protein conformational switches can present on/off biomolecular recognition ability that can be triggered to release or capture another protein on demand, in response to external stimuli such as ion concentration ${ }^{14}$ or temperature. ${ }^{15-17}$

Protein conformational switches are particularly relevant for the design of switchable surfaces and interfaces with programmable functionalities. ${ }^{16}$ Smart and functional materials that can be switched 
remotely will be increasingly used in applications beyond drug delivery and they have been already applied to control cell adhesion, ${ }^{18}$ assemble nanomaterials on surfaces, ${ }^{19}$ control biomolecular interactions for analytical devices ${ }^{15}$ and set the on/off state of bio-valves between catalytic nanoscale compartments. ${ }^{20}$

We aimed to devise a binary protein complex capable of spontaneous self-assembly, on-demand disassembly in response to physical stimulus and re-assembly (Figure 1). We draw inspiration from neuronal SNARE complex, comprising three proteins: Syntaxin, SNAP25, and VAMP (Figure 2A). ${ }^{21}$ The complex assembles via the formation of a parallel coiled-coil of four $\alpha$-helices, two contributed by SNAP25 (green in Figure 2A) and one each by Syntaxin and VAMP2 (red and blue respectively in Figure 2A). The two helices of SNAP25 are kept together by a 70 amino acid long linker that spans from the C-terminal of the first helix to the $\mathrm{N}$-terminal of the second (unstructured, not represented in Figure $2 \mathrm{~A}$ and shown as a sketch in Figure 3A). Syntaxin presents extra $\mathrm{N}$-terminal domains with regulatory function and a C-terminal transmembrane domain for anchoring to the cell membrane (both not represented in Figure $2 \mathrm{~A}$ and shown as a sketch in Figure $3 \mathrm{~A}$ ). VAMP2 presents a C-terminal transmembrane domain which, in the living organism, is embedded in the membrane of synaptic vesicles. The domains that assemble into the tight and stable coiled-coil represented in Figure $2 \mathrm{~A}$ are referred to as SNARE motifs: they are 60 amino acids long and behave as unstructured proteins in solution. The SNARE motifs adopt stable $\alpha$-helical structures only upon assembly with the other SNARE domains, driven by the alignment of several hydrophobic layers towards the core of the complex. ${ }^{21}$ The native SNARE proteins retain their ability to form supramolecular complexes ex vivo and in vitro, but being a ternary system, the native SNAREs do not translate easily into biotechnology applications. ${ }^{22-24}$ We attempted to re-engineer two of the three native SNAREs to yield a simple and robust binary protein system. One of the two elements of the new system is capable of oriented and permanent immobilisation at a solid-liquid interface through an added glutathione S-transferase (GST) domain. ${ }^{25} \mathrm{~A}$ new complementary protein was therefore created by combining Syntaxin SNARE domain with that of VAMP using a designer linker made of a sequence of unstructured amino acids. We then tested whether this newly engineered component retained the ability to spontaneously and specifically self-assemble with the immobilised partner. We also tested whether it can be released in response to a local increase of temperature. The disassembly temperature of the engineered complex could be adjusted by as much as $\sim 40^{\circ} \mathrm{C}$ by altering the polypeptide length of a single SNARE domain. ${ }^{24}$ To illustrate the thermo-responsive release of immobilised recombinant proteins from solid surfaces we tested two distinct polypeptide "tags" and showed that the immobilised tagged proteins are released selectively in a temperature dependent manner. The low temperature tag unfolds and disassembles at $\mathrm{Tm}=42.5^{\circ} \mathrm{C}$, whilst the high-temperature tag remains bound up to $\mathrm{Tm}=79.6^{\circ} \mathrm{C}$. 
Importantly, the released tags do not aggregate, making possible the regeneration of the interface. The low-temperature immobilisation system enables the decoration of dynamically switchable biointerfaces and it is suitable for the immobilisation of proteins or enzymes that can be released on demand by a mild local increase of the temperature.

\section{Results and discussion}

\section{Reductionist re-engineering of ternary SNARE complexes yields temperature- sensitive protein assembly}

First, we used minimised SNARE helixes and their native ternary SNARE protein architecture to check our hypothesis that thermal stability of the tetrahelical assembly can be regulated by adjusting just one of the $\alpha$-helixes. Recombinant SNAP25, Syntaxin and VAMP2 (VAMP2-L) were expressed and purified in E. coli and were used to characterise the thermal stability of the full-length SNARE complex in solution (Figure 2). To adjust the thermal stability of the complex while preserving the structural features that allow self-assembly, a shortened VAMP2 was synthesised (VAMP2-S). Whereas both VAMP2-L and Syntaxin were designed to span the entire SNARE motif (54 amino acids), VAMP2-S was only 25 amino acids long, with the C-terminal truncated just before the ionic layer. A rationale for the truncated design is presented in Supplementary Figure 1.

The assembly of SNARE proteins mixtures was analysed using Synchrotron Radiation Circular Dichroism (SRCD) (Figure 2B). This was enabled by the fact that individual SNARE proteins are unstructured in solutions whereas the ternary complex formed by SNAP25, Syntaxin and VAMP2 is a highly structured coiled-coil. The far-UV SRCD spectra of both complexes containing SNAP25, Syntaxin and either VAMP2-L or VAMP2-S revealed $\alpha$-helical structure. VAMP2-L complex yielded a more intense CD signal at the same molar concentration of VAMP2-S complex, consistent with longer structured $\alpha$-helical domains. Unstructured individual SNARE proteins and binary mixtures from control tests (Supplementary Figure 2) confirmed that the minimalist, engineered, ternary SNARE complexes retain fundamental properties of the native SNARE complex, i.e, the ability of SNARE domains to interact tightly only when four of them form a helical bundle. ${ }^{26}$

Temperature stability of the assembled protein complex was strongly affected by the length of the VAMP2 fragment used. Whilst both VAMP2-L and VAMP2-S complexes showed fully folded $\alpha$-helical structure at $25^{\circ} \mathrm{C}$, their stability at elevated temperatures was clearly very different. Melting temperature of the VAMP2-S complex was reduced compared to that of VAMP2-L complex by $\sim 37^{\circ} \mathrm{C}$ (Figure 2C). 
The results of Figure 2 informed the design of SNARE-derived immobilisation "tags" that can respond to different ranges of temperatures, with VAMP2-L and VAMP2-S suitable to be used for high- and low-temperature release respectively. The full potential of an immobilised protein system would benefit from being binary rather than ternary, so that one protein could be immobilised on a surface of interest and the thermo-responsive tag for controlled release fused to a protein of interest. ${ }^{22}$ To retain the thermal stability properties of the full-length and shortened SNARE complex, while reducing it to a binary system, we extensively re-engineered the native SNAREs by generating chimeras comprising sequences from all three proteins (Figure 3A). VAMP2-L (V-L) and VAMP2-S (V-S) were fused to Syntaxin $(S)$ in tandem and separated by the polypeptide linker natively found between the helixes of SNAP25 protein, to allow the assembly of these two helixes in a parallel configuration (Figure 3B). The new VS recombinant proteins were engineered to have either 54 amino acid domain (VS-L) or 25 amino acid long domain (VS-S), and they were successfully expressed in E. coli and purified.

$\mathrm{HH}$ was engineered to contain shortened SNARE domains from SNAP25 so that the $\alpha$-helices would be precisely 54 amino acids long and consistent with the longest domains of VS-L and VS-S. Since the native domain architecture of $\mathrm{HH}$ remained unchanged, we also retained the native linker domain (Figure 3A) connecting the two domains. The new HH was successfully expressed in bacteria.

To assess the assembly properties of the new binary SNARE mimics we used SRCD and we found that $\mathrm{HH}$ assembles with either VS-L or VS-S to form $\alpha$-helices similarly to their ternary counterpart (Figure 3C). The far-UV SRCD spectra typical of $\alpha$-helices were observed only where the pairs were combined, whereas no $\alpha$-helices were detected with individual proteins, suggesting that the new fusions are also unstructured in solution unless assembled in a complex with specific partners, which is a feature inherited from native SNAREs (Supplementary Figure 4). The melting temperature of the full-length and shortened binary complexes was studied using SRCD temperature scans and the values obtained were $79.9^{\circ} \mathrm{C}$ and $43.3^{\circ} \mathrm{C}$ for HH/VS-L and HH/VS-S respectively (Figure 3D), which are very similar to the values obtained for the corresponding ternary complexes. To prove that the stability of the complex depends on the truncation of the $\mathrm{V}$ domain rather than the nature of the linker domain, we tested an entirely different linker, made of glycine and serine residues only, to connect $\mathrm{V}$ with $\mathrm{C}$ terminal S domains. Far-UV SRCD spectra and temperature scans of the new full-length VS-L2 confirmed that there was no substantial difference between SV-L and SV-L2 (Supplementary Figure 5). Together, these results confirm that the new binary protein complexes retain the ability to selfassemble in solution and possess thermal stability properties similar to that of the ternary protein complexes. 
HH was immobilised on porous Sepharose beads and the assembled VS-L and VS-S complexes' stability was studied at different temperatures. Sepharose beads derivatised with glutathione (GSH) were used to facilitate immobilisation of HH via a recombinant Glutathione S-Transferase (GST) tag fused at the $\mathrm{N}$-terminal of $\mathrm{HH}$ (Figure 4A). The high affinity and remarkable stability of the GST/GSH interaction, combined with the high solubility of the GST tag, makes this system suitable for robust immobilisation of recombinant proteins on GSH-modified resins or other surfaces, such as plates, biosensors, magnetic beads. As GST also has high affinity to gold, this can also be used for direct decoration of gold nanoparticles. ${ }^{25}$

The ability of the GST-HH modified surface to specifically capture VS-L and VS-S was assessed using a pull-down experiment. This was performed using Sepharose beads functionalised with either GST only (negative control) or GST-HH. Upon incubation with VS followed by extensive washing, Sepharose-GST was unable to bind VS-L or VS-S, whereas Sepharose-GST-HH could distinctively pull-down VS-L and VS-S, suggesting specific binding via HH/VS interaction and no background binding contributed by the GST tag or the surface (Figure 4B). As SDS-PAGE only provides a qualitative or semi-quantitative estimation of the amount of protein captured by the beads, VS-L and VS-S were fluorescently labeled to allow quantification of the captured protein. Labeling was achieved by chemical cross-linking Cy5maleimide to the thiol group of a cysteine residue deliberately introduced between the $\mathrm{V}$ domain and the flexible linker of both VS-L and VS-S (Supplementary Figure 6). Sepharose-GST-HH was incubated with an excess of fluorescent VS (VS-L-Cy5 and VS-S-Cy5), similarly to the pull-down described above. This time, fractions of the Sepharose-GST-HH/VS complexes were incubated at a set temperature and unbound protein removed using a washing buffer at the same incubation temperature. The residual Cy5 fluorescence intensity measured on the Sepharose beads represents the proportion of VS still bound to the surface at any tested temperature. The results of the pull-down performed at different temperatures show that both VS-L-Cy5 and VS-S-Cy5 are released from the resin upon incubation at $80^{\circ} \mathrm{C}$. However, a substantial proportion of VS-L-Cy5 remained bound to the surface at $65^{\circ} \mathrm{C}$, whilst the bound VS-S-Cy5 dropped steeply and virtually none was still bound to the beads at the same temperature, with the majority released below $50^{\circ} \mathrm{C}$ (Figure 4C). The reduction of fluorescence intensity observed between $20^{\circ} \mathrm{C}$ and $35^{\circ} \mathrm{C}$ is due to the well documented temperature dependence of fluorescence quantum yield of carbocyanide dyes. ${ }^{27}$

To test whether the system can work like an ideal thermo-responsive switch and capture another payload after a release cycle, an alternative payload was synthesised by conjugation of Cy3 to VS-S (VS-S-Cy3, Supplementary Figure 7). The Sepharose-GST-HH beads were first exposed to VS-S-Cy3, 
then heated at $50^{\circ} \mathrm{C}$ for $20^{\prime}$, washed and exposed to VS-S-Cy5 after cooling at $20^{\circ} \mathrm{C}$. The beads were imaged using confocal microscopy and successful exchange of payload was confirmed by the sharp change of fluorescence emission (Figure 4D).

The ability of Sepharose-GST-HH/VS-S-Cy5 system to preserve capture and release properties upon several cycles of heating at $50^{\circ} \mathrm{C}$ and cooling at $20^{\circ} \mathrm{C}$ was tested for up to 4 cycles (Figure 4E). The results show no substantial changes in the behaviour of the interface, most likely due to the unstructured nature of SNARE-derived peptides that do not aggregate even at higher temperatures. We also tested the ability of Sepharose-GST-HH/VS-L-Cy5 system to withstand cycles of heating at $80^{\circ} \mathrm{C}$ and cooling at $20^{\circ} \mathrm{C}$, and we observed that a significant amount of fluorescence intensity was lost after one cycle, but the remaining constructs were stable over 5 cycles. We attributed this to partial desorption of the incompletely folded recombinant GST portion of the recombinant protein at higher temperatures (Supplementary Figure 8). The correctly folded GST remains on beads and no further change in fluorescence is detectable. This is also consistent with the trend of the VS-L-Cy5 release observed in Figure $4 \mathrm{C}$, which increases at a higher rate above $50^{\circ} \mathrm{C}$, although still exhibiting a marked difference with the release trend of the shorter VS-S-Cy5.

Finally, we tested the release rate of Sepharose-GST-HH beads loaded with VS-S-Cy3 at three different temperatures: 20,37 and $45^{\circ} \mathrm{C}$. At the lowest temperature, only background intensity of $\mathrm{Cy} 3$ fluorescence was measured in the buffer surrounding the beads after 25 minutes. A mild increase of VS-S-CY3 release was observed at $37^{\circ} \mathrm{C}$ (body temperature), suggesting that a delivery system based on this technology would have only moderate off-target loss of the payload. The release at $45^{\circ} \mathrm{C}$ (hyperthermia temperature) was instead substantial within the first 10 minutes and was complete after 20 minutes, suggesting sustained payload release happens above the engineered threshold temperature (Figure 5).

The pull-down results and release curves together confirmed that, upon immobilisation, the thermoresponsive HH/VS interaction preserves the interesting thermal stability properties found in solution and, therefore, can be used as an effective way to release proteins from a surface in response to a local temperature increase. No irreversible protein aggregation was observed, unlike other common protein-protein interactions for which extensive optimisation is required to limit aggregation propensity. ${ }^{28}$ The refolding ability of proteins at the interface and the regeneration of the surface following subsequent cycles of capture and release was tested successfully, suggesting that the interactions involved are fully reversible and therefore compatible with dynamic and programmable surfaces involving biomolecules. ${ }^{16}$ 
Importantly, informed engineering of the coiled-coil structure of the neuronal SNARE complex allowed modulation of the disassembly temperature down to physiologically attainable conditions for in vivo applications. Research into stimuli-responsive nanomaterials suggested many approaches to engineering environmentally controlled molecular systems. Temperature and $\mathrm{pH}$ are the two stimuli most physiologically relevant to drug delivery and release applications. Such systems are typically based on temperature-sensitive spontaneously aggregated or polymerised molecules which undergo depolymerisation or disassembly stimulated by the change in the external conditions. One group of applications rely on spontaneously formed hydrogels which entrap typically small molecule drugs for delayed, sustained or environmentally dependent release. ${ }^{29,30}$ Another category relies on micellar delivery systems which are typically used for the delivery of poorly soluble low molecular weight (LMW) therapeutics and for sustained drug delivery in diverse scenarios, including controlled delivery of multiple drugs for synergistic effect, as elegantly exemplified by Emamzade et al. ${ }^{31}$ Another common approach relies on self-assembled nanoparticles, optionally with added stimuli sensitivity, such as Redox or enzyme sensitive nanoparticles. ${ }^{32-35}$ Polymeric carriers are especially suitable for payloads such as conventional organic drugs which are physically entrapped in the polymer networks. Such entrapment is strongly affected by the size and physical properties of the LMW drugs, and typically no defined molecular ratio of polymer to drug could be specified. In contrast, the described system, which relies on engineered interacting proteins, includes defined molar ratio of the captured protein to the molecular anchor and is likely unaffected by the size or nature of the protein payload. The high specificity and affinity of the interaction between the two protein components exceeds that of typical polymers by far and the stimuli-sensitivity of their interaction can be engineered in a rational manner as described in this paper. Another key distinction is that our system allows multiple rounds of reassembly, whilst hydrogels, micelles and nanoparticle-based systems assume single use; whilst that may not have implications in therapeutic applications, the reversibility of the protein interface provides additional advantages for biotechnology applications. Importantly, the proteinaceous nature of the SNAREs mimic-based system reported here makes it particularly suitable for the thermoresponsive release of proteins, thus expanding the use of thermo-responsive drug delivery towards the vast arsenal of bio-therapeutics. We envisage that combining the thermo-responsive capabilities described here with designer nanocarriers will be especially useful for the development of hyperthermia-directed protein therapeutics. ${ }^{36}$ Elsewhere, peptide-lipid hybrid nanoscale thermoresponsive vesicles were tested in vivo and successful release of doxorubicin following hyperthermia treatment was observed. ${ }^{37}$ Magnetic hyperthermia, which makes use of iron oxide nanoparticles to induce local increase of temperature, is clinically approved in Europe for the treatment of 
glioblastoma ${ }^{38}$ and it represents a promising approach for magneto-responsive local release of therapeutics, towards which the thermo-responsive protein interface described here may contribute.

The use of thermo-responsive, nanoparticle-based systems for local delivery is not limited to magnetic materials activated by alternate magnetic fields but, for example, nanomaterials with the ability to extensively absorb energy in the near-infrared (NIR) spectrum can be used to trigger a localised and controlled increase of temperature in vivo, as radiation in this window has minimal absorbance by tissues and can penetrate to a depth of micrometers to centimeters, making photo-thermal drug release possible. ${ }^{2}$ Dedicated nanomaterials such as gold nanorods with a strong absorbance in the NIR range have been developed and their ability to trigger conformational changes on gold-conjugated biomolecules in response to NIR irradiation has been previously reported in vitro and in vivo. ${ }^{39,40} \mathrm{We}$

272 believe that the thermo-responsive protein release tag properties reported here make it a suitable 273 interface for magneto-responsive and photo-thermal therapy.

\section{Experimental}

\section{Cloning, protein expression, purification and analysis}

DNA inserts were purchased as synthetic genes (Dundee Cell Products) and introduced into pGEX-KG GST gene fusion system (Addgene). The resulting sequences are reported in Supplementary Table 1 and secondary structure data are presented in Supplementary Figures 2 and 3. All plasmids were expressed in BL21(DE3)pLysS E.coli strain (Thermo Fisher Scientific); the expressed recombinant proteins were purified by affinity chromatography using Glutathione Sepharose 4B resins (GE Healthcare). The GST tag was removed by thrombin cleavage, with the exception of GST and GST-HH for which the tag was preserved. Fluorescently labeled proteins were incubated with an excess of Cy5or Cy3-maleimide at this stage. All proteins were further purified by size exclusion chromatography using ÄKTA Pure chromatography system (GE Healthcare) equipped with a Superdex 75 10/300 GL column. All proteins were stored and analysed in $100 \mathrm{mM} \mathrm{NaCl}, 20 \mathrm{mM}$ HEPES pH 7.3. Protein concentration was determined using BCA assay (Thermo Fisher Scientific). SDS-PAGE was performed using 12\% RunBlue SDS protein gels (Expedeon), stained using InstantBlue (Expedeon) and imaged Biosciences) was used for visualisation of fluorescently labeled protein. Quantification of fluorescent 
Far UV SRCD spectra were recorded at the B23 beamline for SRCD of the Diamond Light Source $(U K)^{41}$ using protein solutions at the concentrations indicated in the figure legends diluted in $25 \mathrm{mM} \mathrm{NaCl}, 5$ mM HEPES pH 7.3 in a rotating cylindrical cuvette with $0.2 \mathrm{~mm}$ optical path (Starna) at $20^{\circ} \mathrm{C}$. SRCD spectra of thermal unfolding were recorded from the same solutions in the $25-95^{\circ} \mathrm{C}$ temperature range with an interval of $2.5^{\circ} \mathrm{C}$. Temperature was raised at the rate of $1^{\circ} \mathrm{C}$ per minute and the solutions were allowed to equilibrate for $2 \mathrm{~min}$ before collecting the spectra. The thermal unfolding curves were plotted by selecting the data points at $222 \mathrm{~nm}$ over the range of temperatures scanned. ${ }^{42}$ The melting temperature $\left(T_{m}\right)$ of the complexes was obtained by interpolation of the temperature at which half of the signal was lost. All SRCD spectra were processed using the software CDApps. ${ }^{43}$

\section{Protein pull-down and controlled release}

$5 \mu \mathrm{M}$ GST-HH and $7.5 \mu \mathrm{M}$ VS were incubated at room temperature for 2 hours with Glutathione Sepharose $4 \mathrm{~B}$ beads (GE Healthcare) under constant agitation. Excess protein was removed by repeated centrifugation and washing using $100 \mathrm{mM} \mathrm{NaCl}, 20 \mathrm{mM}$ HEPES pH 7.3. Total protein load was assessed following complete denaturation in hot RunBlue LDS sample buffer (Expedeon) followed by SDS-PAGE using 12\% RunBlue SDS protein gels (Expedeon), stained using InstantBlue (Expedeon) and imaged using a ChemiDoc imaging system (Biorad). Controlled release was obtained by incubation of the GST-HH/VS beads at a set temperature for 20 minutes, followed by a wash at the same incubation temperature. The beads for confocal imaging were prepared in the same way, but exposed to VS-S-Cy3 first and to VS-S-Cy5 after release at $50^{\circ} \mathrm{C}$ and cooling at $20^{\circ} \mathrm{C}$. Repeated heating and cooling cycles were performed on 5 different sets of solutions, each subjected to 0 to 4 cycles as described for the pull-down above before analysis of retained protein was performed. To obtain release curves, Sepharose-GST-HH beads were loaded with VS-S-Cy3 using the same approach above. Three fractions were diluted 5 times and incubated at 20,37 and $45^{\circ} \mathrm{C}$. The beads were quickly sedimented by centrifugation every 5 minutes and the concentration of VS-S-Cy3 released into the buffer was assessed. Quantification of retained fluorescent protein or protein fluorescence released in solution was conducted using an Infinite 200 Pro plate reader (TECAN).

\section{Confocal microscopy}

Images were obtained using a Leica TCS SP8 confocal microscope (20X objective) from VS-S-loaded Sepharose-GST-HH beads dispersions on a glass coverslip. False colour scanning images were obtained using $552 \mathrm{~nm}$ laser excitation and 554-630 nm emission window (Cy3 channel) and $638 \mathrm{~nm}$ laser and 650-750 nm emission (Cy5 channel). 


\section{Conclusions}

324

325

326

327

328

329

330

331

332

333

334

335

336

337

338

339

340

341

342

343

344

345

346

347

348

349

350

351

The results reported here show that modulation of the release temperature of engineered SNARE complexes can be achieved by changing the length of just one SNARE motif which could also be fused to Syntaxin SNARE motif to form a binary interaction complex. The engineered protein interface can be expressed by recombinant means and its thermo-responsive properties adjusted within a broad range of temperatures in the $40-80^{\circ} \mathrm{C}$ range. To fully exploit the potential of this new protein interface, the immobilisation on surfaces other than GSH-crosslinked Sepharose beads should be explored further. For example, GST could be replaced by available peptides with affinity to specific materials or the SNAREs directly linked to the surface using available chemical bioconjugation methods. ${ }^{44,45}$ Once fused to thermo-responsive VS proteins, other recombinant proteins could be potentially immobilised and released from $\mathrm{HH}$-modified materials, irrespective of $\mathrm{HH}^{\prime}$ s immobilisation method. On the other hand, $\mathrm{HH}$-activated materials could be used for the immobilisation and release of any VS-tagged recombinant protein, highlighting the modularity and flexibility of the system described here. It is likely that thermo-responsive SNARE-derived tags will have a role in future design of interfaces with programmable, protein-mediated functions

\section{Conflicts of interest}

There are no conflicts to declare.

\section{Acknowledgements}

We appreciate the technical support of Giuliano Siligardi, Rohanah Hussain, Tamas Javorfi and Charlotte Sarah Hughes from Diamond Light Source (Didcot, UK) and Karen Staines from the University of Lincoln, UK. We thank Diamond Light Source for experimental beamtime on B23 for SM12555, SM15232 and SM20209 sessions.

\section{References}

1. Y. Lu, W. Sun and Z. Gu, J. Control. Release, 2014, 194, 1-19.

2. B. P. Timko, T. Dvir and D. S. Kohane, Adv. Mater., 2010, 22, 4925-4943.

3. P. M. Mendes, Chem. Soc. Rev., 2008, 37, 2512-2529.

4. S. Mura, J. Nicolas and P. Couvreur, Nat. Mater., 2013, 12, 991-1003.

5. H. Tang, H. Kobayashi, Y. Niidome, T. Mori, Y. Katayama and T. Niidome, J. Control. Release, 2013, 171, 178-183. 
6. S. A. Stanley, J. E. Gagner, S. Damanpour, M. Yoshida, J. S. Dordick and J. M. Friedman, Science (80-. )., 2012, 336, 604-608.

7. Koguma, S. Yamashita, S. Sato, K. Okuyama and Y. Katakura, J. Chromatogr. A, 2013, 1305, 149-153.

8. B. J. Nehilla, J. J. Hill, S. Srinivasan, Y.-C. Chen, T. H. Schulte, P. S. Stayton and J. J. Lai, Anal. Chem., 2016, 88, 10404-10410.

9. Y. Jung, H. Bayley and L. Movileanu, J. Am. Chem. Soc., 2006, 128, 15332-15340.

10. L. D. Knecht, N. Ali, Y. Wei, J. Z. Hilt and S. Daunert, ACS Nano, 2012, 6, 9079-9086.

11. K. Chockalingam, M. Blenner and S. Banta, Protein Eng. Des. Sel., 2007, 20, 155-161.

12. M. Nakano, J.-R. Shen and K. Kamino, Biomacormolecules, 2007, 8, 1830-1835.

13. B. Ciani, E. G. Hutchinson, R. B. Sessions and D. N. Woolfson, J. Biol. Chem., 2002, 277, 10150-10155.

14. B. Bulutoglu, K. Dooley, G. Szilvay, M. Blenner and S. Banta, ACS Synth. Biol., 2017, 6, 17321741.

15. J. Hyun, W.-K. Lee, N. Nath, A. Chilkoti and S. Zauscher, J. Am. Chem. Soc., 2004, 126, 73307335.

16. L. Li, N. K. Li, Q. Tu, O. Im, C.-K. Mo, W. Han, W. H. Fuss, N. J. Carroll, A. Chilkoti, Y. G. Yingling, S. Zauscher and G. P. López, Biomacromolecules, 2018, 19, 298-306.

17. J. D. Kim, Y. J. Jung, C. H. Woo, Y. C. Choi, J. S. Choi and Y. W. Cho, Colloids Surfaces $B$ Biointerfaces, 2017, 149, 122-129.

18. M. A. Cole, N. H. Voelcker, H. Thissen and H. J. Griesser, Biomaterials, 2009, 30, 1827-1850.

19. Sun and J. Lahann, Soft Matter, 2009, 5, 1555.

20. Edlinger, T. Einfalt, M. Spulber, A. Car, W. Meier and C. G. Palivan, Nano Lett., 2017, 17, 5790-5798.

21. R. Jahn and R. H. Scheller, Nat. Rev. Mol. Cell Biol., 2006, 7, 631-643.

22. E. Ferrari, F. Darios, F. Zhang, D. Niranjan, J. Bailes, M. Soloviev and B. Davletov, J. Nanobiotechnology, 2010, 8, 9.

23. F. Darios, D. Niranjan, E. Ferrari, F. Zhang, M. Soloviev, A. Rummel, H. Bigalke, J. Suckling, Y. Ushkaryov, N. Naumenko, A. Shakirzyanova, R. Giniatullin, E. Maywood, M. Hastings, T. Binz and B. Davletov, Proc. Natl. Acad. Sci., 2010, 107, 18197-18201.

24. E. Ferrari, M. Soloviev, D. Niranjan, J. Arsenault, C. Gu, Y. Vallis, J. O’Brien and B. Davletov, Bioconjug. Chem., 2012, 23, 479-484.

25. W. Ma, A. Saccardo, D. Roccatano, D. Aboagye-Mensah, M. Alkaseem, M. Jewkes, F. Di Nezza, M. Baron, M. Soloviev and E. Ferrari, Nat. Commun., 2018, 9, 1489. 
26. K. Wiederhold and D. Fasshauer, J. Biol. Chem., 2009, 284, 13143-13152.

27. W.-T. Liu, J.-H. Wu, E. S.-Y. Li and E. S. Selamat, Appl. Environ. Microbiol., 2005, 71, 6453-7.

28. K. Dudgeon, R. Rouet, I. Kokmeijer, P. Schofield, J. Stolp, D. Langley, D. Stock and D. Christ, Proc. Natl. Acad. Sci. U. S. A., 2012, 109, 10879-10884.

29. Y. Chen, Y. Gao, L. da Silva, R.P. Pirraco, M. Ma, L. Yang, R.L. Reis and J. Chen, Polym. Chem., 2018, 9, 4063-4072.

30. T. Sun, C. Zhu and J. Xu, Soft matter, 2018, 14, 921-926.

31. M. Emamzadeh, D. Desmaële, P. Couvreur and G. Pasparakis, J. Mater. Chem. B, 2018, 6, 2230-2239.

32. M. Liu, H. Du, A.R. Khan, J. Ji, A. Yu and G. Zhai, Carbohydr. Polym., 2018, 184, 82-93.

33. L. Zhang, Y. Wang, X. Zhang, X. Wei, X. Xiong and S. Zhou, ACS Appl. Mater. Interfaces, 2017, 9, 3388-3399.

34. Y. Li, H. Hu, Q. Zho. Wan, Y. Wan, H. Xu, Z. Li and X. Yang, ACS Appl. Mater. Interfaces, 2017, 9, 19215-19230.

35. Q. Tang, L. Gao, H. Cong, N. Song, C. Lu, Curr. Med. Chem., 2018, 25, 1837-1866.

36. J. Andrew Mackay and A. Chilkoti, Int. J. Hyperth., 2008, 24, 483-495.

37. Z. S. Al-Ahmady, W. T. Al-Jamal, J. V. Bossche, T. T. Bui, A. F. Drake, A. J. Mason and K. Kostarelos, ACS Nano, 2012, 6, 9335-9346.

38. N. Lee, D. Yoo, D. Ling, M. H. Cho, T. Hyeon and J. Cheon, Chem. Rev., 2015, 115, 1063710689.

39. C. C. Chen, Y. P. Lin, C. W. Wang, H. C. Tzeng, C. H. Wu, Y. C. Chen, C. P. Chen, L. C. Chen and Y. C. Wu, J. Am. Chem. Soc., 2006, 128, 3709-3715.

40. S. Charan, K. Sanjiv, N. Singh, F.-C. Chien, Y.-F. Chen, N. N. Nergui, S.-H. Huang, C. W. Kuo, T.C. Lee and P. Chen, Bioconjug. Chem., 2012, 23, 2173-2182.

41. R. Hussain, T. Jávorfi and G. Siligardi, J. Synchrotron Radiat., 2012, 19, 132-135.

42. S. Laera, G. Ceccone, F. Rossi, D. Gilliland, R. Hussain, G. Siligardi and L. Calzolai, Nano Lett., 2011, 11, 4480-4.

43. R. Hussain, K. Benning, D. Myatt, T. Javorfi, E. Longo, T. R. Rudd, B. Pulford and G. Siligardi, J. Synchrotron Radiat., 2015, 22, 862-862.

44. L. Sola, A. Gori, M. Cretich, C. Finetti, C. Zilio and M. Chiari, in Peptide Microarrays, eds. M. Cretich and M. Chiari, Springer New York, New York, NY, 2016, pp. 167-182.

45. K. E. Sapsford, W. R. Algar, L. Berti, K. B. Gemmill, B. J. Casey, E. Oh, M. H. Stewart and I. L. Medintz, Chem. Rev., 2013, 113, 1904-2074. 
Figure 1. Schematic of the reversible, thermo-responsive protein release system. (A) Reversible temperature sensitive release of a payload (e.g., therapeutic protein) in response to heat activation of a molecular thermal switch $(T)$ which opens at temperatures above $\sim 45^{\circ} \mathrm{C}$; the system can be loaded again by dropping the temperature below the threshold. (B) Engineering of the molecular thermal switch: the self-assembling SNARE proteins ternary complex has been re-engineered into a new binary protein complex capable of sequence specific self-assembly. Tuning the temperature stability of the complex is achieved by modifying the interface region $(T)$. The complex re-assembles if cooled to below the temperature threshold.

Figure 2. Length of SNARE motif affects thermal stability of the SNARE complex. (A) Structural representation of the SNARE complex (based on PDB ID: 1SFC). The structures highlighted in colour indicate the portions of SNARE motifs that have been used in this work: blue indicates 54 or 25 amino acid long VAMP2 motifs, Syntaxin protein is highlighted in red and SNAP25 motifs in green. The parts of the ribbons highlighted in yellow correspond to the ionic layer. (B) Far-UV SRCD spectra of VAMP2$L$ and VAMP2-S complexes, each molecule at $2 \mu \mathrm{M}$ concentration, $\mathrm{pH}=7.3,20^{\circ} \mathrm{C}$. The peak at $222 \mathrm{~nm}$ is highlighted and indicates the wavelength that was used to quantify unfolding and disassembly shown in panel C. (C) CD temperature scan of VAMP2-L and VAMP2-S SNARE complex. The data represent unfolding expressed as \% of increase of CD signal at $222 \mathrm{~nm}$ over a value of 100 extrapolated to infinite temperature. The melting temperature $\mathrm{Tm}$ is derived by fitting the data to a sigmoid and represents the temperature value at which $50 \%$ unfolding is achieved.

Figure 3. Engineering of a binary SNARE complex with altered thermal stability. (A) Schematic of the native SNARE proteins and their domains, used as building blocks for the engineering of binary artificial complexes. Colour code: blue $=$ domain V from VAMP2, red $=$ domain $\mathrm{S}$ from Syntaxin, green $=$ linker and the two SNARE domains from SNAP25. Cylinders represent $\alpha$-helixes, the yellow rectangle represents the membrane into which the SNARE proteins are embedded via an $\alpha$-helical transmembrane domain (VAMP2 and Syntaxin) or palmitoylated cysteine residues (4 zig-zag segments on the SNAP25). The amino acid numbers of the domains effectively used for the engineering of the binary complexes are displayed near the coloured domains, which also correspond to the colour coded highlights of Figure 2A. (B) Schematic of the recombinant SNARE mimics. Cylinders represent SNARE domains highlighted in panel $A$, whereas the green lines correspond to the naturally occurring linker between the two $\alpha$-helices of SNAP25 with four cysteine residues mutated into alanines (orange dots, also highlighted by arrows in the $\mathrm{HH}$ sketch) and one introduced cysteine residue exposing a free thiol 
complexes $\left(8 \mu \mathrm{M}\right.$ of each molecule, $\mathrm{pH}=7.3,20^{\circ} \mathrm{C}$ ) and $(\mathrm{D})$ temperature scans have been obtained as in Figure 2 and resulted in very similar assembly and disassembly properties.

454 Figure 4. Assembly of binary SNARE complexes on GSH activated Sepharose beads and thermoresponsive release of VS. (A) Schematic of $\mathrm{HH}$ immobilisation strategy via GST/GSH interaction. The structure of GST is based on PDB ID 1UA5. (B) SDS-PAGE of the pull-down of VS-L and VS-S by functionalised Sepharose beads. Lanes 1 and 2 show eluates from Sepharose-GST incubated with VSL and VS-S respectively: for both lanes, only a band corresponding to GST was visible (28.6 kDa, indicated by the arrow on the right), suggesting that there is no specific interaction of VS with GST or the surface. Lanes 3 and 4 show eluates from Sepharose-GST-HH incubated with VS-L and VS-S respectively: besides the bands corresponding to GST-HH protein ( $45.3 \mathrm{kDa}$, indicated by the arrow), one intense extra band per lane was detected, corresponding to the mass of VS-L protein $(21.3 \mathrm{kDa}$,

463 lane 3) and VS-S protein (17.2 kDa, lane 4), suggesting specific binding via HH/VS interaction. The lane 464 with the protein marker (PM) allows comparison of the relative mass of the proteins loaded on the SDS-PAGE (relative masses of the standards are listed on the left). (C) Residual fluorescence intensity from Sepharose-GST-HH bound VS-L-Cy5 and VS-S-Cy5 after incubation at different temperatures. Data points and error bars represent the average of three measurements and standard error respectively. (D) Confocal microscopy of representative Sepharose-GST-HH beads exposed to VS-SCy3 first (upper panels), regenerated by heating and exposed to VS-S-Cy5 (lower panels). Left and right panels show the fluorescence at the Cy3 and Cy5 emission wavelengths respectively. Scale bars are 50 $\mu \mathrm{m}$. (E) Fluorescence intensity of captured VS-S-Cy5 after Sepharose-GST-HH was heated and cooled for 0 to 4 cycles. Average of three measurements, error bars represent the standard error.

473 Figure 5. Release of VS-S-Cy3 into the buffer upon incubation at three representative temperatures of loaded Sepharose-GST-HH beads. 
Figure 1

A
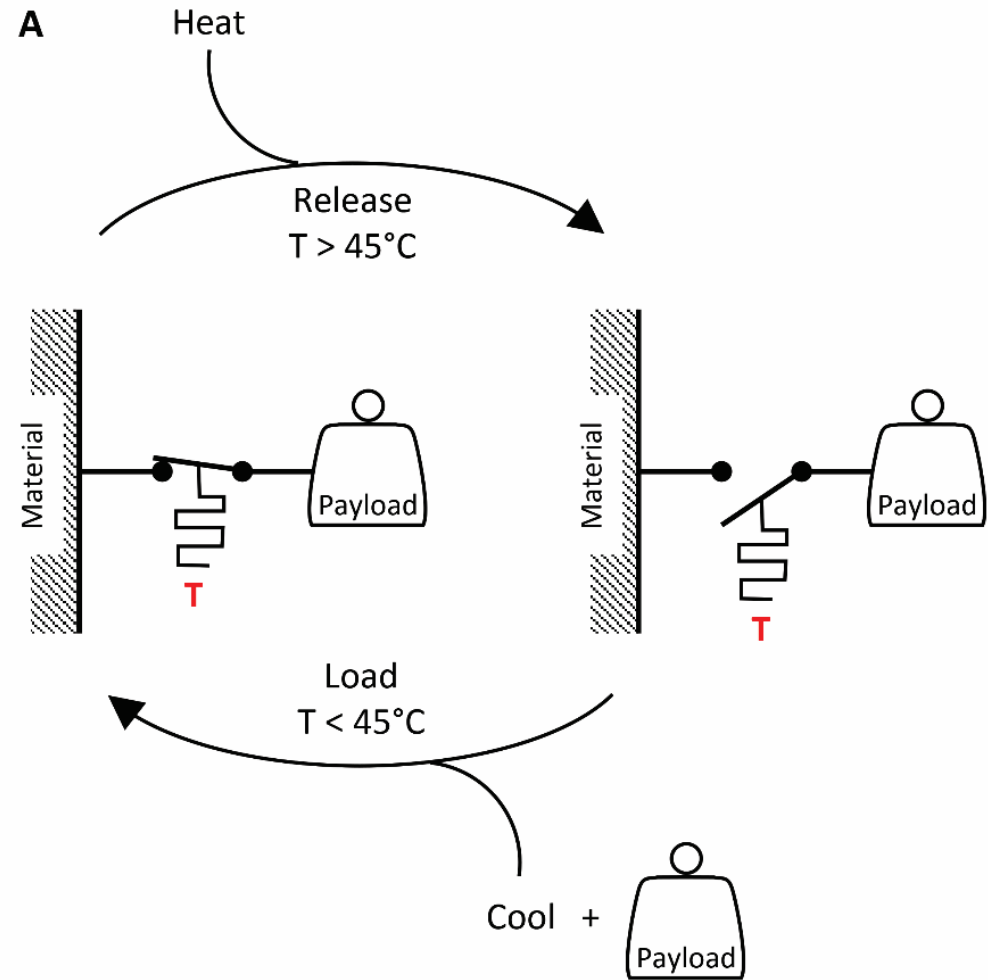

B

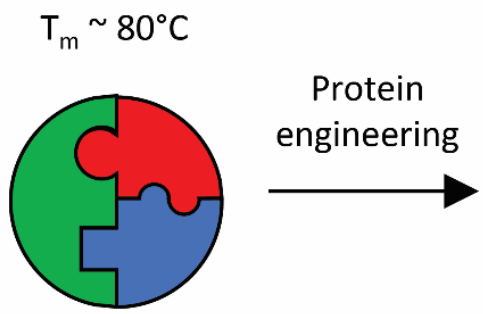

Native synaptic SNARE

proteins ternary arrangement

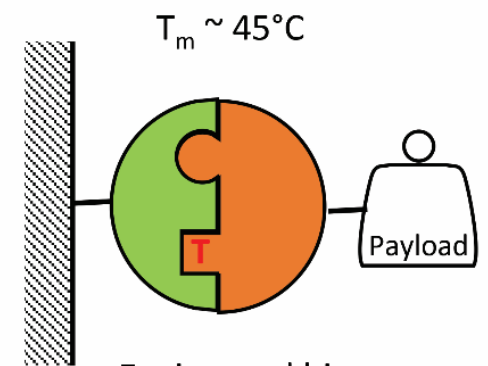

Engineered binary protein-protein interface 
Figure 2

A

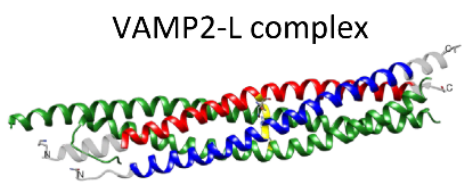

VAMP2-S complex

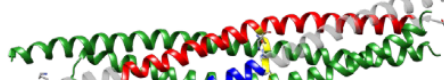

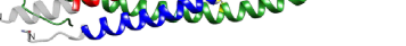

B

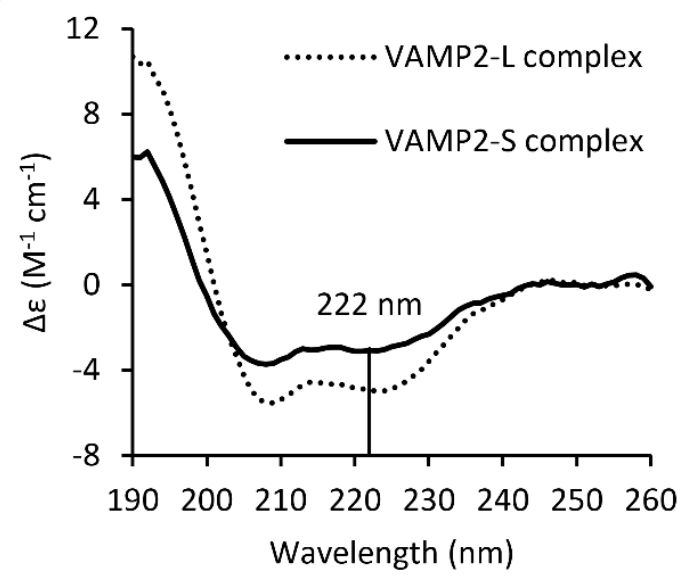

C

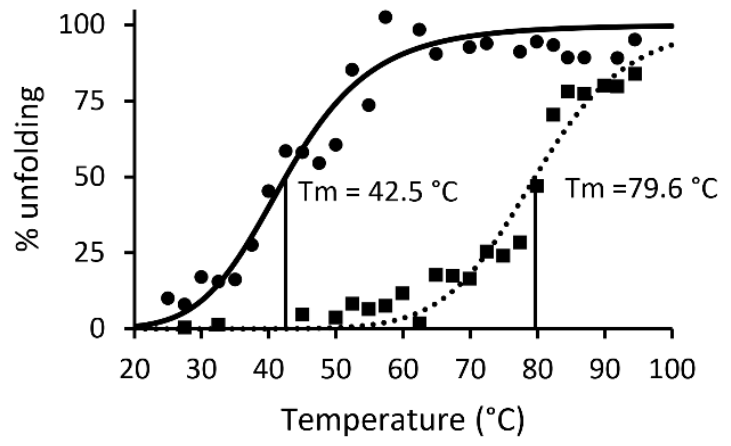


Figure 3

A

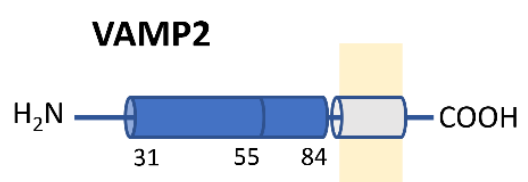

Syntaxin

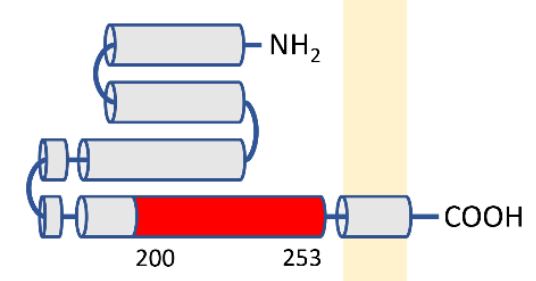

SNAP25

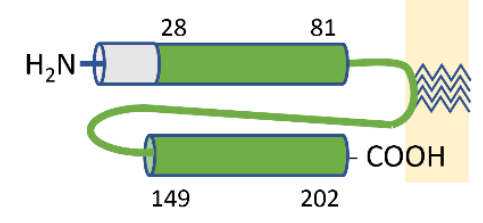

C

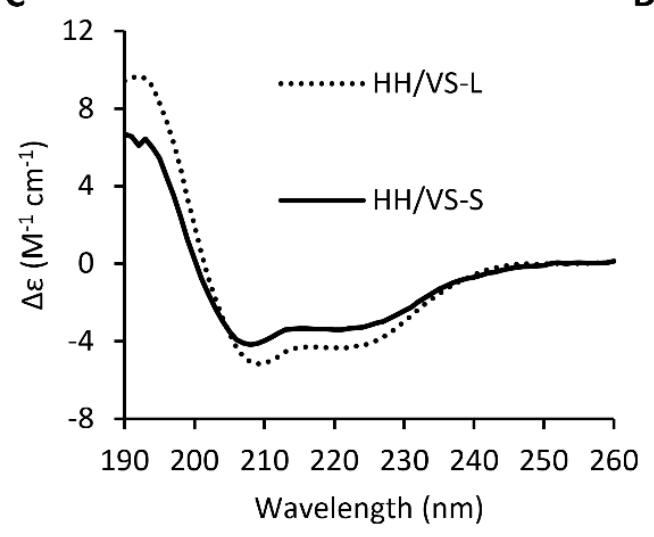

D
B
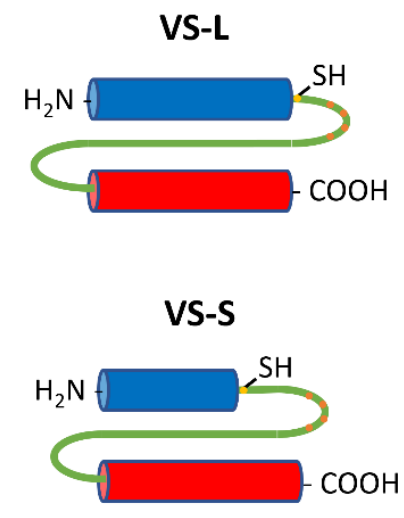

HH

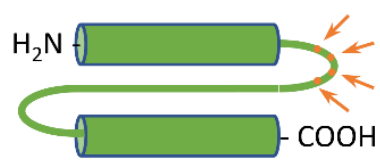

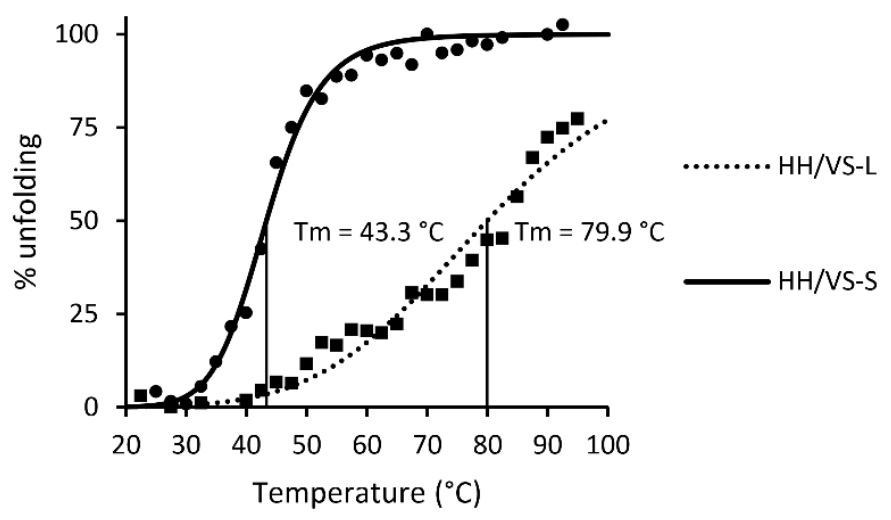


Figure 4

A
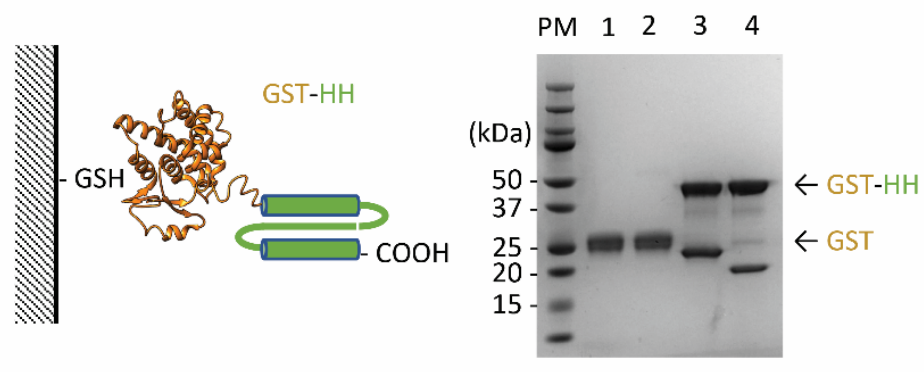

D

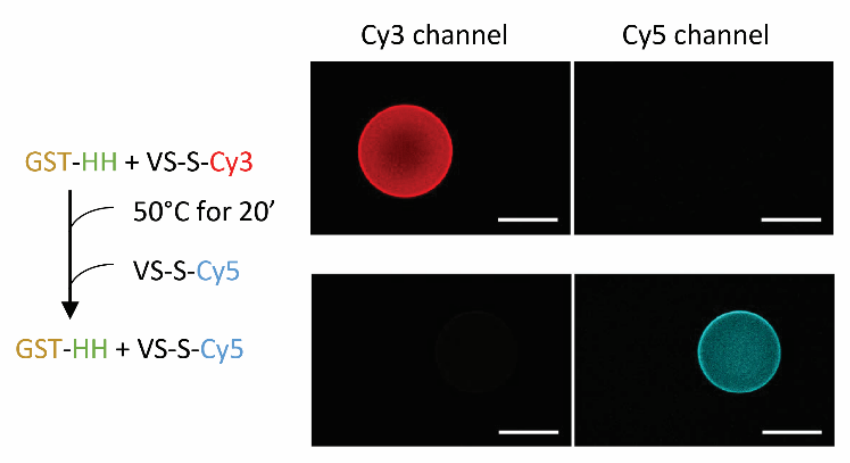

C

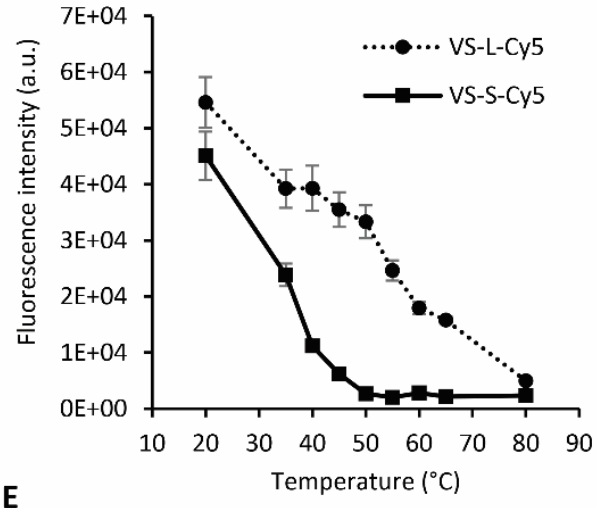

E

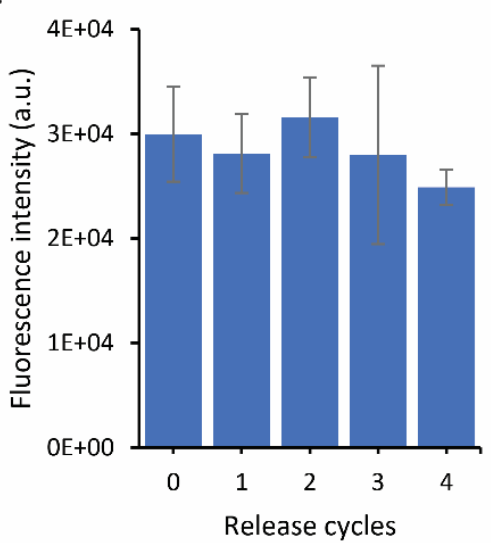


Figure 5

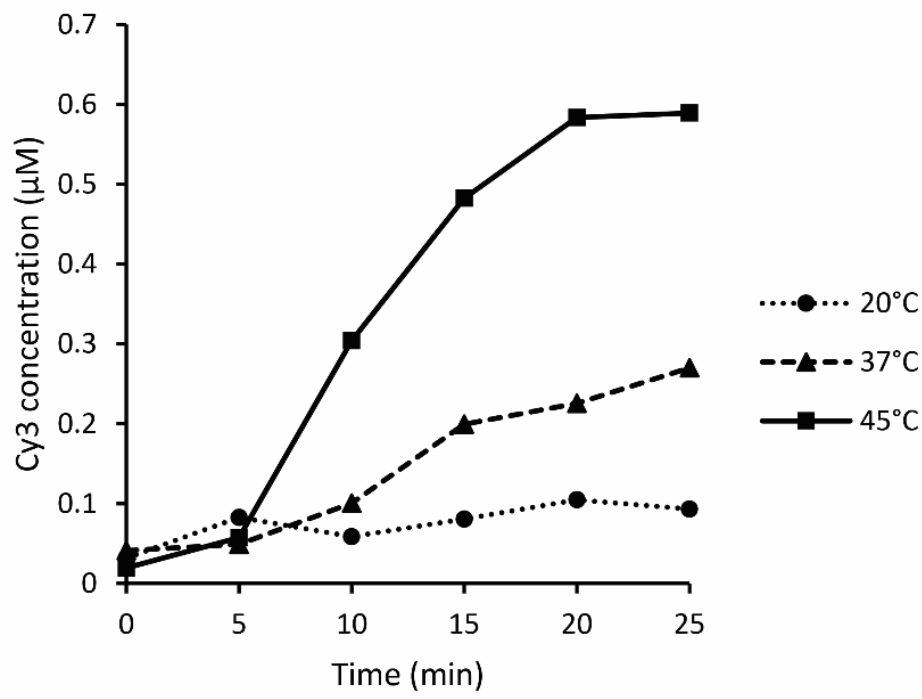

